\title{
Der Rückgabeanspruch eines anderen Vertragsstaats des UNESCO-Kulturgutübereinkommens nach § 6 Abs. 2 KultGüRückG
}

\author{
Oberverwaltungsgericht Nordrhein-Westfalen, Urteil vom 8. Juli 2013 - 5 A 1370/12
}

1. Ein Rückgabeanspruch eines anderen Vertragsstaats des UNESCO-Kulturgutübereinkommens nach §6 Abs. 2 KultGüRückG setzt voraus, dass das zurückgeforderte Kulturgut unrechtmäßig nach dem 26. April 2007 aus dem Hoheitsgebiet des Vertragsstaats in das Bundesgebiet verbracht worden ist. Hierfür genügt nicht, dass die Einfuhr in das Bundesgebiet nach diesem Zeitpunkt stattgefunden hat.

2. Die Beurteilung, dass archäologische Gegenstände vor der unrechtmäßigen Verbringung aus einem Vertragsstaat unbekannt waren, setzt die nähere Kenntnis voraus, unter welchen Umständen sie entdeckt worden sind und in wessen Besitz sie sich seitdem befunden haben. (Amtliche Leitsätze)

\section{Tatbestand}

- Die Klägerin betreibt ein Auktionshaus und wendet sich gegen eine Anhalteanordnung des beklagten Bundeslandes nach dem Kulturgüterrückgabegesetz zur Sicherung der Rückgabe präkolumbischer archäologischer Kunstgegenstände, deren unrechtmäßige Ausfuhr aus Mexiko die Beigeladene geltend macht.

Der ausführliche Sachverhalt kann in der KUR 3/4-2012, $141 \mathrm{ff}$. nachgelesen werden. Dort ist das erstinstanzliche Urteil des Verwaltungsgerichts Köln vom 25. April 2012 - 10 K 3537/11 abgedruckt (Anm. der Redaktion).

\section{Entscheidungsgründe}

Die Berufung hat keinen Erfolg. Das Verwaltungsgericht hat der Klage zu Recht stattgegeben. Die Anhalteanordnung des Beklagten vom 17. Juni 2011 ist rechtswidrig und verletzt die Klägerin in ihren Rechten (§ 113 Abs. 1 S. 1 VwGO).

I. Die Voraussetzungen für die Anhaltung von Kulturgütern nach § 8 Abs. 2 Kulturgüterrückgabegesetz vom 18. Mai 2007 (BGBI. I S. 757) - KultGüRückG - liegen nicht vor. Nach dieser Bestimmung ordnen die für die Rückgabe des Kulturgutes zuständigen Behörden die Anhaltung von Kulturgut an, bei dem der dringende Verdacht besteht, dass es unrechtmäßig aus einem anderen Mitgliedstaat der Europäischen Union oder Vertragsstaat in das Bundesgebiet verbracht worden und an diesen Staat herauszugeben ist. Vertragsstaat ist gemäß $\S 1$ Abs. 1 und 2 KultGüRückG jeder Staat, der das UNESCO-Übereinkommen vom 14. November 1970 über Maßnahmen zum Verbot und zur Verhütung der rechtswidrigen Einfuhr, Ausfuhr und Übereignung von Kulturgut (BGBI. 2007 II S. 626) - Kulturgutübereinkommen - ratifiziert hat, ihm beigetreten ist oder es angenommen hat. Mexiko hat das Kulturgutübereinkommen am 4. Oktober 1972 angenommen, Deutschland hat es am 30. November 2007 ratifiziert.
Die Voraussetzungen der im Rahmen von $\S 8$ Abs. 2 KultGüRückG zu prognostizierenden Rückgabepflicht gegenüber einem Vertragsstaat ergeben sich aus $\S 6$ Abs. 2 S. 1 KultGüRückG. Danach ist ein unrechtmäßig nach dem 26. April 2007 aus dem Hoheitsgebiet eines Vertragsstaats in das Bundesgebiet verbrachter Gegenstand dem Vertragsstaat auf sein Ersuchen zurückzugeben, wenn

1. dieser Gegenstand vor der Verbringung oder im Fall von archäologischen Gegenständen, die vor der Verbringung unbekannt waren, innerhalb eines Jahres, nachdem die zuständige Behörde des betroffenen Vertragsstaats von dem Gegenstand Kenntnis erlangen konnte, von dem ersuchenden Vertragsstaat aus religiösen oder weltlichen Gründen als für die Archäologie, Vorgeschichte, Geschichte, Literatur, Kunst oder Wissenschaft besonders bedeutsam bezeichnet wurde oder ein Verfahren zur Bezeichnung eingeleitet und die Einleitung des Verfahrens öffentlich bekannt gemacht wurde und

2. der Gegenstand einer der in Art. 1 des Kulturgutübereinkommens genannten Kategorien angehört.

Gemäß § 6 Abs. 2 S. 2 KultGüRückG gilt als „besonders bedeutsam bezeichnet" im Sinne von S. 1 Nr. 1 ein Gegenstand, wenn er individuell identifizierbar von einem anderen Vertragsstaat in ein Verzeichnis des bedeutenden öffentlichen und privaten Kulturgutes aufgenommen worden ist. Das Verzeichnis muss im Bundesgebiet ohne unzumutbare Hindernisse öffentlich zugänglich sein. Lässt sich nicht klären, ob ein Gegenstand, der vor dem 26. April 2007 als besonders bedeutsam im Sinne von S. 1 Nr. 1 bezeichnet worden ist, vor oder nach diesem Tag ins Bundesgebiet verbracht worden ist, so gilt er als nach diesem Tag ins Bundesgebiet verbracht.

Ein als „,besonders bedeutsam bezeichnetes“ Kulturgut muss gemäß Art. 1 Kulturgutübereinkommen unter anderem folgenden Kategorien angehören: 
c) Ergebnisse archäologischer Ausgrabungen (sowohl vorschriftsmäßiger als auch unerlaubter) oder archäologischer Entdeckungen;

d) Teile künstlerischer oder geschichtlicher Denkmäler oder archäologischer Stätten, deren Zusammenhang zerstört ist;

e) Antiquitäten, die mehr als hundert Jahre alt sind, wie Inschriften, Münzen und gravierte Siegel;

f) Gegenstände von ethnologischem Interesse

g) Gut von künstlerischem Interesse wie

$[\ldots]$

ii) Originalwerke der Bildhauerkunst und der Skulptur aus einem beliebigen Material

\section{$[\ldots]$.}

§ 6 Abs. 4 KultGüRückG bestimmt, dass Kulturgut unrechtmäßig aus einem anderen Staat verbracht worden ist, wenn bei seiner Ausfuhr gegen die dort gültigen Rechtsvorschriften für den Schutz von Kulturgütern verstoßen worden ist.

II. Gemessen an den Maßstäben des § 6 Abs. 2 S. 1 und S. 2 KultGüRückG und Art. 1 Kulturgutübereinkommens besteht nicht der dringende Verdacht, dass die angehaltenen Artefakte an die Beigeladene herauszugeben sind. Es deuten keine konkreten Anzeichen darauf hin, die Gegenstände könnten unrechtmäßig nach dem 26. April 2007 aus dem Hoheitsgebiet eines Vertragsstaats in das Bundesgebiet verbracht worden sein (dazu unten 1.). Darüber hinaus fehlen Gesichtspunkte, die die Beurteilung zulassen, dass es sich um im Sinne von $\S$ 6 Abs. 2 S. 1 Nr. 1 KultGüRückG nachträglich eintragungsfähige "unbekannte" Kulturgegenstände handelt (dazu unten 2.). Mit einer Ausnahme fehlt es bei sämtlichen übrigen angehaltenen Gegenständen an weiteren Voraussetzungen für einen Rückgabeanspruch nach § 6 Abs. 2 KultGüRückG (dazu unten 3.). Schließlich besteht auch nicht der dringende Verdacht, dass der Beigeladenen zivilrechtliche Herausgabeansprüche aus Eigentum zustehen, die die Anhalteanordnung rechtfertigen könnten (dazu unten 4.).

1. Es deuten keine konkreten Anzeichen darauf hin, dass die angehaltenen Objekte unrechtmäßig nach dem 26. April 2007 aus dem Hoheitsgebiet eines Vertragsstaats in das Bundesgebiet verbracht worden sind. § 6 Abs. 2 KultGüRückG setzt nach Wortlaut [dazu unten a)], Systematik [dazu unten b)] und Entstehungsgeschichte [dazu unten c)] voraus, dass sowohl die Ausfuhr aus dem Herkunftsstaat als auch die Einfuhr in das Bundesgebiet nach dem 26. April 2007 erfolgt sind. Für eine unrechtmäßige Ausfuhr nach dem 26. April 2007 bestehen keine Anhaltspunkte [dazu unten d)]. a) Nach dem eindeutigen Wortlaut des $\S 6$ Abs. 2 KultGüRückG ist entscheidend, dass das unrechtmäßige Verbringen aus dem Hoheitsgebiet eines Vertragsstaats in das Bundesgebiet (vollständig) nach dem 26. April 2007 stattgefunden haben muss. Die Aufnahme des Datums in den Gesetzestext dient der Beschränkung der innerstaatlichen Regelungen zum Kulturgutübereinkommen auf zukünftige Sachverhalte nach dem Inkrafttreten des Vertragsgesetzes in Deutschland, weil eine Rückwirkung verfassungsrechtlich als äußerst problematisch angesehen wurde (vgl. Gesetzentwurf der Bundesregierung, BT-Drs. 16/1371, S. 1, 6, 13, 22, iVm dem Gesetz zu dem Übereinkommen vom 14. November 1970 über Maßnahmen zum Verbot und zur Verhütung der rechtswidrigen Einfuhr, Ausfuhr und Übereignung von Kulturgut vom 20. April 2007 (BGBI. II S. 626)).

Hätte der Gesetzgeber entsprechend der Auffassung des Beklagten und der Beigeladenen für die Regelung zukünftiger Sachverhalte lediglich auf die Einfuhr nach Deutschland abstellen wollen, hätte sich dies in einem entsprechend abweichenden Gesetzeswortlaut niederschlagen müssen. Hierfür hätte sich etwa angeboten, die Rückgabepflicht auf unrechtmäßig aus einem Vertragsstaat ausgeführte und nach dem 26. April 2007 in das Bundesgebiet verbrachte Gegenstände zu erstrecken.

b) Systematisch wird die Wortlautauslegung dadurch bestätigt, dass $\S 6$ Abs. 2 KultGüRückG bewusst $\S 6$ Abs. 1 KultGüRückG nachgebildet ist, der die Richtlinie 93/7/EWG des Rates vom 15. März 1993 (ABI. L 74/74) umsetzt (vgl. Gesetzentwurf der Bundesregierung, BT-Drs. 16/1371, S. 18).

Nach ihrem Art. 13 gilt diese Richtlinie vorbehaltlich einer nach Art. 14 Abs. 2 zulässigen abweichenden Regelung nur in Fällen, in denen Kulturgüter ab dem 1. Januar 1993 unrechtmäBig aus dem Hoheitsgebiet eines Mitgliedstaats verbracht werden (vgl. Rietschel, aaO, S. 94).

Es spricht nichts dafür, dass im Gegensatz hierzu der Ausfuhrzeitpunkt aus dem Herkunftsstaat für die nach diesem Vorbild gefasste Regelung in $\S 6$ Abs. 2 KultGüRückG bedeutungslos sein sollte.

c) Schließlich bestätigt entstehungsgeschichtlich die vom Gesetzgeber angestrebte und völkerrechtlich gebotene Orientierung am Kulturgutübereinkommen die Wortlautauslegung des $\S 6$ Abs. 2 KultGüRückG, wonach der gesamte Vorgang der Verbringung aus dem Herkunftsstaat in das Bundesgebiet nach dem 26. April 2007 stattgefunden haben muss. Bei deutscher Beteiligung ist das Übereinkommen nicht vor diesem Zeitpunkt für beide Staaten rechtsverbindlich geworden. Dementsprechend kommt eine völkerrechtsfreundliche Auslegung zu keinem anderen Ergebnis.

aa) Der Gesetzgeber hat sich bewusst an den Verpflichtungen aus dem Kulturgutübereinkommen ausgerichtet. Dabei hat er sowohl „eine zu laxe und damit rufschädigende als auch [...] 
eine zu strenge und damit nicht mehr praktikable Umsetzung" des Übereinkommens vermieden. Dementsprechend hat er das Gesetz als Ergebnis einer Abwägung der Belange des Kulturgutschutzes auf nationaler und internationaler Ebene sowie des Kunsthandels angesehen (vgl. Gesetzentwurf der Bundesregierung, BT-Drs. 16/1371, S. 12 (linke Spalte unten) und S. 13 (rechte Spalte, 2. Absatz)).

Gerade die Voraussetzungen für eine Rückgabepflicht in $\S 6$ Abs. 2 KultGüRückG sind nach der Gesetzesbegründung (S. $18 \mathrm{f}$.) an Formulierungen des Kulturgutübereinkommens angepasst. Dadurch sollte eine ausufernde und missbräuchliche Ausweitung von Rückgabeansprüchen auf eine Vielzahl weniger bedeutsamer Kulturgüter vermieden werden. Die Vorschrift knüpft deshalb an die individualisierbare Eintragung der Kulturgüter in ein öffentlich zugängliches Verzeichnis im Sinne von Art. 5 Buchstabe b) Kulturgutübereinkommen an. Danach verpflichten sich die Vertragsstaaten zur Einrichtung von Dienststellen, deren Aufgabe unter anderem die Aufstellung und Führung eines Verzeichnisses des bedeutsamen öffentlich und privaten Kulturguts ist, dessen Ausfuhr für das nationale kulturelle Erbe einen merklichen Verlust bedeuten würde.

bb) Das Kulturgutübereinkommen selbst entfaltet gleichfalls - entsprechend der völkervertragsrechtlichen Regel in Art. 28 des Wiener Übereinkommens über das Recht der Verträge (BGBI. 1985 II S. 926) - keine Rückwirkung (vgl. im Ergebnis ebenso Rietschel, aaO, S. 24 mwN).

Danach binden vorbehaltlich abweichender Vereinbarungen völkervertragliche Bestimmungen eine Vertragspartei nicht in Bezug auf eine Handlung oder Tatsache, die vor dem Inkrafttreten des Vertrags hinsichtlich der betreffenden Vertragspartei vorgenommen wurde oder eingetreten ist, sowie in Bezug auf eine Lage, die vor dem genannten Zeitpunkt zu bestehen aufgehört hat. In diesem Sinne enthält das Kulturgutübereinkommen keine Pflichten zur Rückgabe von Kulturgut, das vor Inkrafttreten des Übereinkommens für die betreffenden Staaten aus dem Ursprungsland entfernt worden ist. Das folgt bereits aus Art. 15 Kulturgutübereinkommen. Danach bleibt bezogen auf früher ausgeführtes Kulturgut lediglich Raum für bereits geschlossene oder neue Sonderabkommen zwischen einzelnen Vertragsstaaten. So wird die völkerrechtlich gebotene Gegenseitigkeit gewährleistet. Derartige bilaterale Zusatzabkommen hat Deutschland nicht abgeschlossen (vgl. Gesetzentwurf der Bundesregierung, BT-Drs. 16/1371, S. 13).

Dementsprechend lassen sich auch Art. 7 Buchstabe b) Kulturgutübereinkommen keine Pflichten der Vertragsstaaten entnehmen, fremdes Kulturgut zurückzugeben, das vor Inkrafttreten des Übereinkommens in den betreffenden Staaten aus dem Ursprungsland entfernt worden ist. Nach Untergliederungspunkt i) müssen die Vertragsstaaten die Einfuhr von Kulturgut verbieten, das nach Inkrafttreten des Übereinkommens für die betreffenden Staaten aus einem Museum, einem religiösen oder weltlichen öffentlichen Baudenkmal oder einer ähnlichen
Einrichtung in einem anderen Vertragsstaat gestohlen worden ist, sofern nachgewiesen werden kann, dass dieses Gut zum Bestand jener Einrichtung gehört. Auch die in Art. 7 Buchstabe b) ii) Kulturgutübereinkommen bezeichnete Pflicht, geeignete Maßnahmen zur Wiedererlangung und Rückgabe von Kulturgut zu ergreifen, bezieht sich nur auf das in Art. 7 Buchstabe b) i) Kulturgutübereinkommen bezeichnete Kulturgut, das nach Inkrafttreten des Übereinkommens für die betreffenden Staaten nachweislich aus einem Museum oder einer ähnlichen Einrichtung im Ursprungsstaat gestohlen und in einen anderen Vertragsstaat eingeführt worden ist. Dementsprechend müssen Diebstahl aus einem Einrichtungsbestand und illegaler Import zeitlich nach dem Inkrafttreten der Konvention in beiden betroffenen Staaten liegen (vgl. Rietschel, aaO, S. 29).

Dies folgt bereits systematisch aus der Regelung betreffend die Rückgabe in einem Untergliederungspunkt von Buchstabe b) des Art. 7 Kulturgutübereinkommen. Zwar kommt in der amtlichen deutschen Übersetzung und in der englischen Fassung durch den unklaren Bezug der Formulierungen "solchen Kulturguts" bzw. „any such cultural property“ nicht zweifelsfrei zum Ausdruck, dass auch in Unterpunkt ii) das unter i) bezeichnete Kulturgut gemeint ist. Dies ergibt sich jedoch sprachlich unmissverständlich aus der gemäß Art. 18 Kulturgutübereinkommen klaren und gleichfalls verbindlichen französischen Fassung. Nach dieser bezieht sich die im Unterpunkt ii) bezeichnete Pflicht, Maßnahmen zur Wiedererlangung und Rückgabe zu ergreifen, auf "tout bien culturel ainsi volé". Diese Fassung präzisiert die gemeinten Kulturgüter dahingehend, dass es sich um "so gestohlene" handeln muss. Damit wird sprachlich zweifelsfrei der Bezug hergestellt zu den im Unterpunkt i) genannten Kulturgütern, die nach dem Inkrafttreten des Übereinkommens für die betreffenden Staaten nachweislich aus dem Bestand eines Museums oder einer ähnlichen Einrichtung gestohlen worden sind. Auch die gleichfalls völkerrechtlich verbindliche spanische Fassung greift mit der Formulierung "todo bien cultural robado" auf, dass es sich um gestohlene Kunstgüter handeln muss, wie sie in Unterpunkt i) bezeichnet sind, und stellt so sinngemäß den Bezug hierzu her.

Die Beschränkung der völkerrechtlich geforderten Maßnahmen zur Rückgabe von Kulturgütern auf solche, die nach Inkrafttreten des Kulturgutübereinkommens in den betreffenden Staaten aus dem Ursprungsland entfernt worden sind, entspricht auch dem allgemeinen Regelungszweck des Übereinkommens. Es dient ausweislich seiner Vorbemerkung unter anderem dem Schutz des im Hoheitsgebiet jedes Vertragsstaats vorhandenen Kulturguts vor den Gefahren des Diebstahls, der unerlaubten Ausgrabung und der rechtswidrigen Ausfuhr, weil die rechtswidrige Einfuhr, Ausfuhr und Übereignung von Kulturgut der Verständigung zwischen den Nationen im Wege steht. Es begründet hingegen keine nachträglichen Rückgabepflichten für Kulturgüter, die vor der völkerrechtlichen Verbindlichkeit des Übereinkommens für die beteiligten Staaten bereits ausgeführt waren. 
Nichts anderes ergibt sich aus Art. 13 Buchstabe d) Kulturgutübereinkommen, der keine direkte Rückgabepflicht begründet. Er verpflichtet im Rahmen der innerstaatlichen Rechtsordnung lediglich dazu, das unantastbare Recht jedes Vertragsstaats anzuerkennen, bestimmtes Kulturgut als unveräußerlich einzustufen und zu erklären, das daher ipso facto nicht ausgeführt werden darf, und die Wiedererlangung solchen Gutes durch den betreffenden Staat in Fällen zu erleichtern, in denen es ausgeführt worden ist (vgl. zur Schwäche dieses Schutzes Rietschel, aaO, S. 39 f.; ähnlich Halsdorfer, IPRax 2008, 395, 397 f.).

Ungeachtet der begrenzten Reichweite der Verpflichtung kann es wegen der fehlenden Rückwirkung des Übereinkommens im Sinne der Gegenseitigkeit nur um eine Anerkennung der Unveräußerlichkeit bestimmter Güter und von hieraus folgenden Ausfuhrverboten für die Zukunft gehen. Dementsprechend muss nur die Wiedererlangung solcher Kulturgüter erleichtert werden, die nach dem Inkrafttreten des Übereinkommens für beide Vertragsstaaten rechtswidrig ausgeführt worden sind. Dies ergibt sich zugleich aus Art. 3 Kulturgutübereinkommen, wonach unter anderem die Ausfuhr nur dann als rechtswidrig gilt, wenn sie im Widerspruch zu den Bestimmungen steht, die von den Vertragsstaaten auf Grund des Übereinkommens angenommen worden sind. Solche Bestimmungen eines Vertragsstaats sind für einen anderen völkerrechtlich erst verbindlich, nachdem das Kulturgutübereinkommen auch für inn in Kraft getreten ist. Dementsprechend muss als Voraussetzung einer rechtswidrigen Ausfuhr im Sinne des Übereinkommens ein später erfolgter Verstoß etwa gegen die durch das Übereinkommen veranlasste Genehmigungspflicht für die vorschriftsmäßige Ausfuhr von Kulturgut oder das zu verhängende Verbot der Ausfuhr von Kulturgut ohne Ausfuhrbescheinigung (Art. 6 Kulturgutübereinkommen) festgestellt werden können.

cc) Der Gesetzesbegründung lässt sich auch im Übrigen nicht entnehmen, dass im Gegensatz zum klaren Gesetzeswortlaut und zur völkerrechtlichen Verpflichtung Deutschlands nur die Verbringung in das Bundesgebiet maßgeblich sein sollte (vgl. ebenso VG Köln im angefochtenen Urteil vom 25. April 2012 - 10 K 3537/11 -, [...], Rn. 41; zustimmend Feddersen, KUR 2012, 125; a.A. VG München, Beschlüsse vom 25. Januar 2010 - M 17 E 09.4878 -, und vom 27. Januar 2010 - M 17 E 09.4833 -, [...], Rn. 15, sowie Rietschel, Internationale Vorgaben zum Kulturgüterschutz und ihre Umsetzung in Deutschland, 2009, S. 147).

Missverständlich ist insoweit die Formulierung, wonach die Bestimmungen des Ausführungsgesetzes wegen der fehlenden Rückwirkung „für die Rückgabe von Kulturgut, das in den vergangenen Jahren im Irak gestohlen und illegal ausgeführt worden ist, überwiegend nicht anwendbar sein [werden], aber künftig illegal nach Deutschland verbrachte Kulturgüter erfassen." (vgl. Gesetzentwurf der Bundesregierung, BT-Drs. 16/1371, S. 13).
Sie lässt offen, ob die Vorschrift auf in den vergangenen Jahren illegal ausgeführte Kulturgüter schon nicht anwendbar sein sollte oder ob nur der Zeitpunkt der Einfuhr nach Deutschland maßgeblich sein sollte.

Die vom VG München in seinem Beschluss vom 27. Januar 2010 - M 17 E 09.4833 - angeführte Begründung auf Seite 18 rechte Spalte, Mitte, des Gesetzentwurfs ist im Ergebnis ebenso wenig ergiebig. Sie bezieht sich auf die Vermutungsregelung in § 6 Abs. 2 S. 4 KultGüRückG, wonach ein Gegenstand, der bereits vor Inkrafttreten des Vertragsgesetzes am 26. April 2007 als besonders bedeutsam iSv S. $1 \mathrm{Nr}$. 1 bezeichnet worden ist, im Zweifel als nach diesem Tag ins Bundesgebiet verbracht gilt. Nur bezogen auf einen derartigen Gegenstand hebt die Begründung die sich aus Satz 1 grundsätzlich ergebende doppelte Voraussetzung der Rückgabepflicht hervor, dass der Zeitpunkt der Verbringung in das Bundesgebiet sowohl nach dem Inkrafttreten des Vertragsgesetzes als auch nach dem Zeitpunkt gelegen haben muss, zu dem der Herkunftsstaat das Kulturgut als besonders bedeutsam bezeichnet oder zumindest das entsprechende Verfahren eingeleitet und seine Einleitung öffentlich bekannt gemacht hat. Dies stimmt schon insoweit mit der Gesetzesfassung nicht überein, als die Vermutungsregelung in Satz 4 die Einleitung eines Eintragungsverfahrens und ihre öffentliche Bekanntmachung vor dem Inkrafttreten des Vertragsgesetzes gerade nicht genügen lässt. Nur für bei Inkrafttreten des Vertragsgesetzes bereits als besonders bedeutsam bezeichnetes Kulturgut gilt auch die der Vermutungsregelung zu Grunde liegende Erwägung (Gesetzesbegründung S. 19), dass dem Besitzer der Nachweis abverlangt werden kann, ob es sich im relevanten Zeitpunkt bereits im Bundesgebiet befunden hat. Überdies fehlt es an jeder Erläuterung, weshalb der Zeitpunkt der Ausfuhr aus dem Herkunftsstaat entgegen dem Wortlaut in Satz 1 in der Begründung als weitere Voraussetzung der Rückgabepflicht nicht einmal erwähnt wird. Auch dies spricht eher für ein Versehen der Entwurfsverfasser als für einen Hinweis auf die gewollte Bedeutungslosigkeit eines gesetzlichen Tatbestandsmerkmals. Insgesamt hinterlässt diese Passage der Begründung für das Verständnis des Gesetzes mehr offene Fragen als sie verlässliche Antworten liefert. Jedenfalls haben danach nicht auszuschließende etwaige abweichende Regelungsabsichten im Gesetzeswortlaut keinen hinreichenden Niederschlag gefunden und sind daher rechtlich nicht maßgeblich.

d) Im Streitfall besteht nicht der gemäß $\S 8$ Abs. 2 KultGüRückG erforderliche dringende Verdacht dafür, die vom Beklagten angehaltenen Gegenstände könnten unrechtmäßig nach dem 26. April 2007 aus dem Hoheitsgebiet der Beigeladenen ausgeführt worden sein. Hierfür gibt es nicht einmal konkrete Anzeichen.

Der Beklagte hat eine Ausfuhr der angehaltenen Kunstgegenstände aus Mexiko nach dem 26. April 2007 in der angefochtenen Verfügung ebenso wenig behauptet wie die Beigeladene in der Verbalnote ihrer Botschaft vom 17. Juni 2011. Die Beigeladene geht vielmehr selbst davon aus, dass es sich um archäolo- 
gische Artefakte aus bisherigem außermexikanischem Privatbesitz handelt. Den dringenden Verdacht, dass die Gegenstände aus nicht autorisierten Grabungen stammen bzw. ihr Fund den zuständigen Behörden nicht gemäß den in Mexiko gültigen gesetzlichen Bestimmungen angezeigt worden ist, leitet die Beigeladene ausschließlich aus dem Erfordernis einer Exportgenehmigung sowie dem Umstand ab, dass nach ihrer Kenntnis entsprechende Genehmigungen für keines der Objekte erteilt worden sind. Damit ist nicht einmal ausgeschlossen, dass die Jahrhunderte alten Gegenstände bereits zu einem Zeitpunkt außer Landes gebracht worden sind, als es noch keine verpflichtenden Ausfuhrgenehmigungen gab. Auch kann eine etwaige Genehmigungserteilung lange zurück liegen und lediglich den heutigen Akteuren nicht mehr bekannt sein. Selbst eine denkbare unrechtmäßige Ausfuhr kann über 30 Jahre zurück liegen, sodass Rückgabeansprüche nach $\S 11$ Abs. 1 S. 3 KultGüRückG erloschen wären.

Obwohl das Rückgabebegehren wegen fehlender Angaben zum Ausfuhrzeitpunkt demnach bereits unschlüssig war, hat die Klägerin zu jedem einzelnen Stück mitgeteilt, von welchem Sammler sie es erhalten hat und wie lange es bereits Gegenstand einer privaten Sammlung außerhalb von Mexiko war, soweit sie die Einlieferer hierzu befragen konnte und diese über eigenes Wissen und einschlägige Unterlagen verfügten. Auf der Grundlage dieser Erkenntnisse hat sie für sämtliche Stücke, zu deren Herkunft sie Näheres wusste, angegeben, dass sie bereits seit Jahrzehnten, jedenfalls vor dem 26. April 2007, Gegenstand außermexikanischer Privatsammlungen waren. Diesen Darlegungen hat der Beklagte in zweiter Instanz vorsorglich widersprochen, ohne für irgendeinen konkreten Gegenstand Gesichtspunkte aufzuzeigen, die auf eine illegale Ausfuhr aus Mexiko nach dem 26. April 2007 hindeuten könnten. Dieses Vorbringen ist unsubstantiiert. Es gibt dem Gericht keine Veranlassung, sozusagen „ins Blaue hinein“, von Amts wegen dem Ausfuhrzeitpunkt weiter nachzugehen. Dies gilt vor allem angesichts des Vorbringens der Klägerin, das auf lange zurück liegende Ausfuhrzeitpunkte hindeutet. Ob die Gegenstände nach dem 26. April 2007 im Rahmen des Kunsthandels aus anderen Ländern nach Deutschland gelangt sind, ist für einen möglichen Rückgabeanspruch der Beigeladenen bedeutungslos, weil es an dem erforderlichen Verbringen aus dem Herkunftsland nach dem maßgeblichen Zeitpunkt fehlt.

Die Vermutungsregelung für Zweifelsfälle in § 6 Abs. 2 S. 4 KultGüRückG reduziert nicht die Darlegungslast des Beklagten. Sie greift nur, wenn sich nicht klären lässt, ob ein Gegenstand, der vor dem 26. April 2007 als besonders bedeutsam im Sinne von S. $1 \mathrm{Nr}$. 1 bezeichnet worden ist, vor oder nach diesem Tag ins Bundesgebiet verbracht worden ist. Damit bezieht sie sich weder auf den Ausfuhrzeitpunkt noch auf Kulturgüter, die - wie hier - erst nach diesem Zeitpunkt als besonders bedeutsam bezeichnet worden sind. Sie gilt nach dem klaren Wortlaut auch nicht für solche archäologischen Gegenstände, die vor der Verbringung unbekannt waren und gemäß § 6 Abs. 2 Nr. 1 KultGüRückG noch innerhalb eines Jahres nach möglicher
Kenntnisnahme nachträglich in ein nationales Kulturgutverzeichnis aufgenommen werden können. Für diese bedarf es des Nachweises der Rückgabevoraussetzungen durch den Herkunftsstaat. Die Vermutungsregel ist bewusst auf Gegenstände beschränkt worden, die bereits vor Inkrafttreten des Vertragsgesetzes am 26. April 2007 als besonders bedeutsam im Sinne von S. $1 \mathrm{Nr}$. 1 bezeichnet worden sind. Nur für diese wurde eine zumutbare Obliegenheit der Besitzer angenommen zu dokumentieren, dass sich die Kulturgüter bereits bei Inkrafttreten des Vertragsgesetzes im Bundesgebiet befunden haben (vgl. Gesetzentwurf, BT-Drs. 16/1371, S. 19).

2. Auch unabhängig vom unklaren Ausfuhrzeitpunkt besteht kein dringender Verdacht, dass die Voraussetzungen für eine Rückgabeverpflichtung nach $\S 6$ Abs. 2 KultGüRückG vorliegen könnten. Es fehlen Gesichtspunkte, die die Beurteilung zulassen, dass es sich um iSv § 6 Abs. 2 S. 1 Nr. 1 KultGüRückG nachträglich eintragungsfähige „unbekannte" Kulturgegenstände handelt. Die von der Anhalteanordnung betroffenen Kunstgegenstände waren nicht vor der Verbringung aus dem Hoheitsgebiet der Beigeladenen in ihr nationales Kulturgüterverzeichnis aufgenommen. Soweit der Beklagte und die Beigeladene geltend machen, es habe sich um unbekannte Gegenstände gehandelt, die nachträglich durch Eintragung in das Verzeichnis als besonders bedeutsam hätten bezeichnet werden können, liegen dafür keine greifbaren Anhaltspunkte vor. Zwischen den Beteiligten besteht Einigkeit, dass die Objekte sämtlich Teile außermexikanischer Privatsammlungen waren. Allein die Tatsache, dass sie aktuellen mexikanischen Behördenvertretern nicht bekannt waren, weil sie zur Zeit der Anhaltung nicht in das nationale Verzeichnis für Kulturgüter eingetragen waren, sagt nichts darüber aus, ob es unbekannte Gegenstände iSv § 6 Abs. 2 KultGüRückG waren. Nicht jeder in einem Verzeichnis fehlende Kunstgegenstand ist bereits unbekannt, nur weil er bei den nationalen Behörden des jeweiligen Vertragsstaats aktenmäßig nicht erfasst ist. Dies kann bei mehrere hundert Jahre alten Gegenständen aus präkolumbischer Zeit auch darauf beruhen, dass sie zu einem früheren Zeitpunkt bekannt waren oder hätten bekannt sein können, der weit vor Inkrafttreten des Kulturgüterschutzrechts gelegen haben kann. Es ist sogar denkbar, dass bewusst oder wegen unzureichender behördlicher Kapazitäten in früheren Jahren von einer Eintragung in das nationale Kulturgüterverzeichnis abgesehen worden ist, obwohl hierzu Gelegenheit bestand. Für solche Fälle greift die Möglichkeit zur nachträglichen Eintragung in ein nationales Register nicht. Ausweislich der Gesetzesbegründung sollten durch den Gesetzesbegriff „archäologische Gegenstände“ unbekannte Bodenfunde sowie Funde aus Raubgrabungen erfasst werden, bei denen keine Gelegenheit für eine frühere Eintragung bestand (vgl. Gesetzesbegründung, BT-Drs. 16/1371, S. 18).

Die Eintragungsmöglichkeit besteht sodann nur innerhalb eines Jahres nach der Möglichkeit der Kenntnisnahme (vgl. hierzu etwa BayVGH, Beschluss v. 13. April 2010 - 7 CE 10.258 - , [...], Rn. 27 f.). 
Ohne nähere Kenntnis, unter welchen Umständen die Kulturgüter entdeckt worden sind und in wessen Besitz sie sich seitdem befunden haben, lässt sich nicht verlässlich beurteilen, ob der Herkunftsstaat zu einem früheren Zeitpunkt von ihnen Kenntnis hatte oder hätte nehmen können. Je länger im nationalen Verzeichnis nicht aufgenommene Gegenstände schon als Sammlerstücke dienen, desto größer ist die Darlegungslast des Herkunftsstaats, wenn er sich darauf beruft, die Kulturgüter seien unbekannt gewesen.

Beweiserleichterungen hat der Gesetzgeber insoweit aus Gründen der Rechtssicherheit im internationalen Kunsthandel in $\S 6$ Abs. 2 S. 4 KultGüRückG nur für Gegenstände vorgesehen, die vor dem 26. April 2007 in ein nationales Verzeichnis für Kulturgüter aufgenommen worden sind (vgl. Gesetzesbegründung, BT-Drs. 16/1371, S. 18 f.).

Wird die Rückgabe anderer Gegenstände beansprucht, muss deshalb bezogen auf den Rückgabeanspruch nach § 6 Abs. 2 KultGüRückG dargelegt und ggf. bewiesen werden, dass keine frühere Möglichkeit bestand, diese Objekte in ein Kulturgüterverzeichnis aufzunehmen. Dies ist etwa dann möglich, wenn eine Raubgrabung oder die Nichtanzeige eines Bodenfundes entdeckt wird und der Nachweis der anschließenden illegalen Ausfuhr gelingt. In Fällen dieser Art lassen sich Rückgabeansprüche selbst hinsichtlich nachträglich in das nationale Kulturgüterverzeichnis aufgenommener Gegenstände gemäß § 6 Abs. 2 S. 1 Nr. 1 KultGüRückG verwirklichen.

Fehlen - wie hier - entsprechende Erkenntnisse über konkrete Raubgrabungen, liegt geradezu der klassische Fall vor, in dem der Gesetzgeber durch Ausgestaltung der Darlegungsund Beweislast dem rechtssicheren Kunsthandel mit nicht in ein öffentlich zugängliches Verzeichnis aufgenommenen Gütern, deren Existenz möglicherweise zu einem früheren Zeitpunkt im Herkunftsstaat einmal bekannt war, den Vorrang gegenüber den Belangen des Kulturgutschutzes eingeräumt hat. Dies wird nicht dadurch in Frage gestellt, dass bei dieser Rechtslage faktisch auch die Rückgabe von archäologischen Gegenständen aus Raubgrabungen der jüngsten Vergangenheit deutlich erschwert sein mag. Ohne erheblichen Ermittlungsaufwand der Herkunftsländer wird sich kaum nachweisen lassen, dass die betreffenden Objekte erst kürzlich entdeckt und illegal außer Landes gebracht worden sind.

Nach der gegenwärtigen Rechtslage besteht gleichwohl kein Raum für eine richterrechtliche Korrektur. Das gilt selbst mit Blick darauf, dass auch die Bundesregierung in ihrem Bericht zum Kulturgutschutz in Deutschland vom 24. April 2013 Handlungsbedarf für eine gesetzliche Nachbesserung sieht, weil die vergleichsweise hohen Rückgabeanforderungen des Kulturgüterrückgabegesetzes bisher von keinem Rückgabe ersuchenden Staat erfüllt werden konnten (vgl. BT-Drs. 17/13378, S. 8 ff.).
Allein dem Gesetzgebers steht eine Entscheidung darüber zu, Rückgabeansprüche anderer Vertragsstaaten künftig anders als bisher ggf. auch unter Hinnahme von Einbußen bei der Rechtssicherheit des Kunsthandels über das völkerrechtlich verpflichtende Maß hinaus auszuweiten, um im diplomatischen Verkehr den gleichwohl erhobenen Vorwurf einer zu restriktiven Handhabung völkerrechtlicher Verpflichtungen zu vermeiden.

3. Abgesehen von dem Auktionsstück mit der Nummer 220, das sich nach Einliefererangaben seit 1987 in Belgien befunden hat und erst nach dem 26. April 2007 nach Deutschland gelangt ist, bestehen für sämtliche übrigen angehaltenen Objekte auch aus anderen Gründen keine Rückgabeansprüche nach $\S 6$ Abs. 2 KultGüRückG. Deshalb kann auf sich beruhen, ob Veröffentlichungen in älteren gedruckten Auktionskatalogen als zumutbare Gelegenheit für den Herkunftsstaat anzusehen waren, von Kulturgütern früher Kenntnis zu nehmen.

a) Soweit die angehaltenen Gegenstände bereits über 30 Jahre vor Erlass der Anhalteverfügung aus dem Hoheitsgebiet der Beigeladenen ausgeführt worden sind, sind etwaige Rückgabeansprüche jedenfalls bereits nach $\S 3$ Abs. 1 S. 3 KultGüRückG erloschen. Die Klägerin hat dies auf der Grundlage von Einliefererangaben für die Objekte geltend gemacht, die in ihrem Katalog unter den Nummern 199, 201-214, 217 und 221 verzeichnet sind. Diesem Vorbringen hat der Beklagte nur vorsorglich, pauschal und ohne jegliche Substantiierung widersprochen.

b) Selbst nach der Rechtsauffassung des Beklagten scheiden Rückgabeansprüche für die Gegenstände aus, die vor dem 26. April 2007 in das Bundesgebiet eingeführt worden sind. Dies hat die Klägerin für die Objekte mit den Katalognummern 200, 201, 216 und 219 unter Bezugnahme auf Angaben der Einlieferer geltend gemacht. Auch diesen Angaben hat der Beklagte nicht substantiiert widersprochen.

Unabhängig davon, ob und gegebenenfalls welche Gegenstände vor der Versteigerung im Juni 2011 zur Vorbesichtigung in Brüssel gewesen sind, bevor sie zur Versteigerung nach Köln zurückgeführt worden sind, kommt es für das Bestehen von Rückgabeansprüchen der Beigeladenen hierauf nicht an. Der Schutz des Kunsthandels für vor dem 26. April 2007 nach Deutschland verbrachte Kulturgüter entfällt nicht nachträglich dadurch wieder, dass sie später ohne Bezug zum Ursprungsland in ein anderes Land gebracht und anschließend wieder zurückgeführt werden. Dieser Sachverhalt stellt kein nach § 6 Abs. 2 KultGüRückG erforderliches Verbringen aus dem rückfordernden Vertragsstaat in das Bundesgebiet dar.

c) Für die Auktionsstücke unter den Katalognummern 222, 223, 225 und 226 ist eine Herkunft aus dem Hoheitsgebiet der Beigeladenen nicht ausreichend belegt. Diesbezüglich sind konkurrierend mit Verbalnote vom 10. Juni 2011 auch von Guatemala Rückgabeansprüche geltend gemacht worden. Die Feststellungen des von der Beigeladenen entsandten Archäologen 
genügen jedoch nicht, um Guatemala als mögliches Herkunftsland auszuschließen. Dieser hat lediglich festgestellt, dass es sich um archäologische Stücke mit charakteristischen Merkmalen der - im Südosten Mexikos angesiedelten - Maya-Kultur aus der klassischen mesoamerikanischen Epoche (200-700 n. Chr.) handelt. Dem hiergegen erhobenen Einwand der Klägerin, die Maya hätten nicht nur in Süd- und Südost-Mexiko, sondern auch in Teilen von Guatemala, Honduras und Belize gelebt, sind der Beklagte und die Beigeladene nicht mehr entgegen getreten. Nähere Aufklärungsmöglichkeiten zum genauen Herkunftsort in der Grenzregion der genannten Staaten sind nicht erkennbar.

d) Ob ein Rückgabeanspruch hinsichtlich des Auktionsstücks mit der Nummer 220 auch deshalb ausscheidet, weil es schon seit 1987 in Belgien war, bevor das Kulturgutübereinkommen dort im Jahr 2009 in Kraft getreten ist, bedarf keiner Klärung. Insoweit besteht jedenfalls - wie oben unter 1. d) für sämtliche angehaltenen Gegenstände ausgeführt - nicht der dringende Verdacht, dass das Objekt unrechtmäßig nach dem 26. April 2007 aus dem Hoheitsgebiet der Beigeladenen in das Bundesgebiet verbracht worden ist.

4. Die Voraussetzungen des $\S 8$ Abs. 2 KultGüRückG sind auch dann nicht gegeben, wenn hierbei zusätzlich denkbare zivilrechtliche Herausgabeansprüche aus Eigentum in den Blick genommen werden, die gemäß § 13 Abs. 6 KultGüRückG auf dem ordentlichen Rechtsweg zu verfolgen sind. Es besteht nicht der dringende Verdacht, dass Rückforderungsansprüche der Beigeladenen aus dem geltend gemachten unveräußerlichen und zeitlich unbegrenzten Eigentum gegeben sein können. Bei Jahrhunderte alten Kunstgegenständen ist bereits denkbar, dass eine wirksame Veräußerung schon erfolgt ist, bevor eine nationale Regelung über unveräußerliches Eigentum in Kraft getreten ist (vgl. etwa OLG Frankfurt, Urteil vom 4. Februar 2013 - 16 U 161/11 -, [...], Rn. 24 ff.).

Darüber hinaus kommt selbst für unveräußerliches Eigentum nach der im internationalen Sachenrecht maßgeblichen lex rei sitae (vgl. § 43 EGBGB) ein gutgläubiger früherer Eigentumserwerb Dritter in außermexikanischen Ländern in Betracht, für die das Kulturgutübereinkommen im Erwerbszeitpunkt nicht oder noch nicht galt oder in deren Rechtsordnung gutgläubiger Erwerb auch an Sachen denkbar ist, an denen ein unveräußerliches Eigentum anderer Staaten besteht (vgl. hierzu näher Palandt-Thorn, BGB, 72. Aufl., 2013, Art. 43 EGBGB, Rn. 5 mwN; OLG Frankfurt, Urteil vom 4. Februar 2013 - 16 U 161/11 -, [...], Rn. 28 ff.; siehe ferner Rietschel, aaO, S. 106 ff.).

Dem steht nicht entgegen, dass in Art. 13 Buchstabe d) Kulturgutübereinkommen das Recht jedes Vertragsstaats anerkannt wird, bestimmtes Kulturgut als unveräußerlich einzustufen und zu erklären, das daher ipso facto nicht ausgeführt werden darf. Diese Anerkennung erfolgt nur im Rahmen der innerstaatlichen Rechtsordnung und steht deshalb unter dem
Vorbehalt einer abweichenden Beurteilung nach den innerstaatlichen Vorschriften über das internationale Sachenrecht (vgl. Halsdorfer, IPRax 2008, 395, 397 f.).

Gemessen daran genügt für die Geltendmachung zivilrechtlicher Ansprüche aus dem Eigentum die bloße Bezugnahme auf eine Bestimmung über das nationale Eigentum an Kulturgütern und ihre Unveräußerlichkeit zumindest dann nicht, wenn gänzlich unklar ist, ob das Eigentum an seit langem im Rechtsverkehr befindlichen Gegenständen entweder bereits vor Inkrafttreten einer solchen Regelung oder danach gemäß den innerstaatlich maßgeblichen Normen einer anderen - völkerrechtlich nicht durch das Kulturgutübereinkommen gebundenen - Rechtsordnung auf einen Dritten übergegangen ist.

So liegt der Fall hier. Trotz der Herkunftsangaben der Klägerin, wonach die angehaltenen Gegenstände schon seit den 50er- oder 70er-Jahren des letzten Jahrhunderts oder später in Belgien, Frankreich, den Vereinigten Staaten von Amerika oder anderen Ländern - teilweise im Rahmen öffentlicher Auktionen - durch Privatsammler erworben worden sind, bleiben Einzelheiten der Erwerbsvorgänge unklar. Mögliche noch frühere Übereignungen bleiben gänzlich im Dunkeln. Aufklärungsmöglichkeiten sind insoweit nicht ersichtlich. (Entscheidung von der Redaktion bearbeitet.) 